\title{
Quantitative Evaluation of Signaling Events in Drosophila S2 Cells
}

\author{
David Bond", David A. Primrose* and Edan Foley" \\ Department of Medical Microbiology and Immunology, University of Alberta, Edmonton AB, Canada T6G 2S2 \\ \#Corresponding Author: Edan Foley, Dept. of Medical Microbiology and Immunology, University of Alberta, Edmonton AB, Canada T6G 2S2. Phone: (780) \\ 492-2303 ; Fax: (780) 492-9828; E-mail: efoley@ualberta.ca \\ *Authors contributed equally.
}

Submitted: September 24, 2007; Revised: November 19, 2007; Accepted: December 9, 2007

Indexing terms: RNA interference, drosophila, immunity

Abbreviations: IKK, I-kappa kinase; Imd, immune deficiency; JNK, c-Jun N-terminal kinase; PGN, gram-negative bacterial peptidoglycan; PGRP-LC, peptidoglycan recognition protein-LC;TNF, tumor necrosis factor

\section{ABSTRACT}

Drosophila activates a robust defense response to gram-negative bacteria through the Immune deficiency (Imd) pathway. Imd signaling proceeds through c-Jun N-terminal Kinase (JNK), NF-kB and caspase modules. The individual signaling modules act in a highly coordinated manner to yield a stereotypical response to infection. While considerable attention has focused on NF-kB-mediated antimicrobial activities, more recent studies have highlighted the involvement of JNK signaling in the Imd pathway response. JNK signaling occurs in a transitory burst and drives the expression of a number of gene products through the AP-1 transcription factor. In this report, we describe a simple method for the quantification of JNK activation by Western blot analysis or directly in tissue culture plates.

\section{INTRODUCTION}

Innate immunity is an ancient and essential first line of defense identifiable in all extant multicellular species (1). Innate immunity is mediated by non-rearranging germline encoded gene products that activate potent antimicrobial defenses. The critical value of this relatively understudied aspect of immunity is underscored by the discovery that components of innate immunity drive adaptive defenses in vertebrates (2). Engagement of innate immune signaling proceeds through conserved signaling pathways, which trigger transcription of host defense genes ranging from antimicrobial peptides to cytokines. For example, the mammalian Tumor Necrosis Factor (TNF) signaling pathway induces a diverse array of physiological responses to the TNF- $\alpha$ cytokine via NF- $\kappa B$, c-Jun N-terminal Kinase (JNK) and caspase signaling modules. A critical event in TNF signaling is mediated by the combinatorial action of the JNK and NF- $\mathrm{KB}$ pathways.
Predominant activation of the JNK pathway ushers in apoptotic death, while a robust NF- $\mathrm{KB}$ response counters JNK-dependent death and commits the cell to survival . Thus, the balance of the two opposing pathways has profound implications for the nature of the immune response, and a breakdown of communication between JNK and NF- $\mathrm{KB}$ signaling has profound implications for host survival. As the signaling pathways that drive innate immune responses are evolutionarily conserved, genetically tractable models have emerged as powerful tools for the identification and characterization of innate immune signaling components (4). For example, the landmark discovery that the Toll pathway drives antifungal defenses in Drosophila prompted the search for and subsequent characterization of mammalian Toll-like receptors (5).

The Drosophila Immune deficiency (Imd) pathway shares numerous similarities with the TNF pathway (6). Engagement of Imd signaling is initiated by the detection 
of gram-negative bacterial peptidoglycan (PGN) by the Peptidoglycan Recognition Protein-LC (PGRP-LC) (7-9). PGRP-LC then signals through the adapter protein Imd (RIP1 ortholog) (10). This results in the dIAP2 and TAB2dependent activation of dTAK1 (TAK1 ortholog) (11-13), which activates both Basket $(14,15)$ (Bsk, JNK ortholog) and the Drosophila I-Kappa Kinase (IKK) complex (16). Bsk phosphorylates and concomitantly triggers the nuclear translocation of Jra (Jun-related antigen, Jun ortholog), which partners with Kayak (Fos ortholog) to initiate the transcription of a subset of the immune responses genes. At the same time IKK-mediated phosphorylation of Relish (Rel, NF- $\kappa B$ ortholog) results in the cleavage and concomitant nuclear translocation of $\operatorname{Rel}(17,18)$. While the exact mechanism of Rel processing requires clarification, the caspase- 8 ortholog Dredd is essential for Rel activation and Rel processing occurs at a consensus caspase clevage site $(19,20)$. Thus, it appears likely that Rel is either directly cleaved by Dredd or a Dredd-responsive caspase. Cleaved Rel rapidly transcribes a large number of gene products that drive the antimicrobial response.

In addition to the overt molecular similarities between the Imd and TNF pathways, there are distinct similarities between the nature of the NF- $\mathrm{BB}$ and JNK-mediated responses. Specifically, JNK signaling is transitory in both cases and this is due in part to NF-kB-mediated suppression of JNK activation. Analagous to the situation in mammals, Rel-dependent transcripts directly contribute to the inactivation of Bsk in Drosophila and loss of either IKK or Rel result in enhanced Bsk signaling (21). A large number of assays exist to quantify Rel-dependent aspects of the Imd pathway; reporter constructs, Northern blot, Western blot, real-time PCR. In contrast, few protocols exist to quantify JNK activation in the Imd pathway. JNK is a member of the MAP kinase family and is activated through phosphorylation by upstream kinases. In this manuscript, we describe a protocol for the visualization and quantification of Bsk activation using commercially available active-JNK antibodies and infrared-based detection methods. The assay is equally applicable in Western blot analysis and for direct quantification of Bsk activation in tissue culture cells. Interestingly, we demonstrate that the In-Cell quantification method is easily extended to quantification of unrelated signaling events.

\section{MATERIALS AND METHODS}

\section{S2 Cell Culture}

S2 cells were maintained at $25^{\circ} \mathrm{C}$ in HyQ TNM-FH medium (Gibco) supplemented with 10\% heat inactivated fetal calf serum, penicillin and streptomycin. Serum free S2 cells were maintained at $25^{\circ} \mathrm{C}$ in HyQ SFX-Insect Serum free medium (Gibco) supplemented with penicillin and streptomycin. For induction of JNK phosphorylation S2 cells were incubated in $50 \mu \mathrm{g} / \mathrm{ml}$ LPS (Sigma) at $25^{\circ} \mathrm{C}$ for the indicated times. For induction of apoptosis $\mathrm{S} 2$ cells were incubated in $10 \mu \mathrm{M}$ Actinomycin D (Sigma) for the indicated times.

\section{Quantification of Apoptosis}

For quantification of apoptosis in S2 cells by flow cytometry, $150 \mu \mathrm{l}$ cells were plated into individual wells of a 96 well plate overnight at a density of $1 \times 10^{6}$ cells per ml. The cells were then incubated with $1 \mathrm{mM} \mathrm{CuSO}_{4}$ to induce expression of Grim and fixed at various times after copper addition overnight at $-20^{\circ} \mathrm{C}$ in $70 \%$ ethanol. The cells were resuspended in PBS containing 30 $\mathrm{gg} / \mathrm{ml}$ Propidium Iodide and $100 \mu \mathrm{g} / \mathrm{ml}$ RNase A (Both Sigma). The cells were incubated for 30min and analyzed by flow cytometry (FACScalibur Becton Dickinson).

\section{Western blotting and protein quantification}

S2 cells were harvested by centrifugation at $1000 \mathrm{Xg}$ for 3 minutes and lysed on ice for 10 minutes in lysis buffer (10mM Tris ( $\mathrm{pH} 7.4), 10 \mathrm{mM} \mathrm{NAH}_{2} \mathrm{PO}_{4}, 150 \mathrm{mM} \mathrm{NaCl}, 1 \%$ Triton X-100). Lysates were spun for 10 minutes at 21000Xg and sample loading buffer was added to the supernatants. Proteins were separated on a $10 \%$ SDSPAGE gel and analyzed by Western blotting. Antiphospho-JNK antibody was purchased from Cell Signaling. Anti-JNK antibody was purchased from Santa Cruz biotechnology. Alexa-fluor 680 and Alexa-fluor 750 secondary antibodies were purchased from Invitrogen. Protein quantification was performed using a Licor Aerius automated infrared imaging system according to manufacturer's instructions.

\section{Plate based protein assay and quantification}

S2 cells were plated in $150 \mu \mathrm{l}$ serum-free medium at one million cells per $\mathrm{ml}$ in 96 well plates and incubated for three days at $25^{\circ} \mathrm{C}$. Cells were fixed in $3.7 \%$ formaldehyde (Sigma) and solubilized in $0.1 \%$ Triton X-100. In Cell quantitative analysis was carried out as described in the protocol section. Briefly, fixed and permeabilized cells were blocked for one hour in blocking buffer. Afterwards, 
cells were incubated with anti-phospho-JNK or active caspase- 3 antibodies overnight at $4^{\circ} \mathrm{C}$. Cells were then washed and incubated with the appropriate Alexa-fluor 750-labeled secondary antibody and alexa-fluor 680coupled phalloidin. After a brief wash step the plates were then visualized using a Licor Aerius automated infrared imaging system according to manufacturer's instructions. Alexa-fluor 680-coupled phalloidin was purchased from Invitrogen. Anti-phospho-JNK was purchased from Cell Signaling. Anti-active-caspase-3 was purchased from AbCam.

\section{RNAi}

Experiments requiring RNAi were performed as described above with the addition of $10 \mu \mathrm{g} / \mathrm{ml}$ dsRNA to each well. dsRNA was generated in an in vitro transcription reaction as described previously (22). Briefly, template DNA was amplified from genomic DNA using gene-specific primers with a GGGCGGGT anchor sequence at the $5^{\prime}$ end. The template DNA was amplified in a second round of PCR with a universal primer bearing the T7 RNA polymerase promoter sequence followed by the anchor sequence (TAATACGACTCACTATAGGGAGACCACGGGCGGGT) . dsRNA was generated from second round PCR products in an in vitro reaction with T7 RNA polymerase. dsRNA was heated to $80^{\circ} \mathrm{C}$ and cooled slowly to room temperature to allow annealing. Gene-specific primer sequences are as follows:

Gene Primer sequence

Kenny1 GGGCGGGTTCAGCGTACTCTTACTGGTCT

Kenny2 GGGCGGGTCACTCGTTTGAGTTCGTACCA

TAK1 GGGCGGGTGAAGTCCACATAGGCTGCCTG

TAK2 GGGCGGGTCACTAATGTATCGATGACGGT

\section{Results and Discussion}

\section{A Western Blot Assay for Quantification of JNK Phosphorylation}

We established a quantifiable Western blot assay to monitor Bsk phosphorylation in the Imd pathway. To induce Imd pathway signaling, we incubated the Drosophila embryonic S2 cell line with a commercial preparation of lipopolysaccharides (LPS). Commercially available LPS are contaminated with PGN and are routinely used to activate Imd pathway signaling in S2 cells (23). We prepared lysates from S2 cells at various Foley et al. - Quantitative Evaluation of Signaling Events in Drosophila S2 Cells. www.biologicalprocedures.com

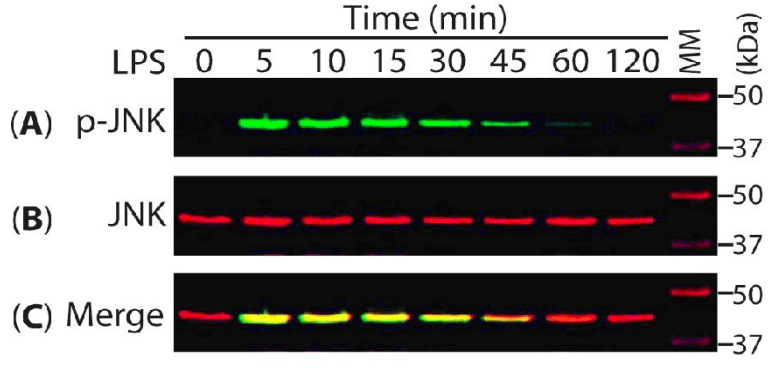

(D)

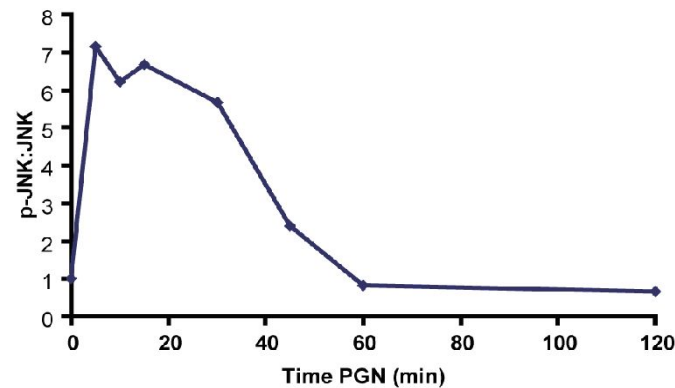

Fig. 1: Time course of Bsk phosphorylation in response to LPS. $(A, B)$ Western blot analysis of total and phospho-Bsk protein (p-Bsk) in S2 cells treated with LPS for the indicated periods. Lysates were probed with anti-p-JNK (A) and anti-JNK (B) antibodies on a single blot. The primary antibodies were detected with Alexa-fluor 680 and Alexa-fluor 750-labeled secondary antibodies, respectively. Panels $A$ and $B$ are false-colored and merged in $C$, with total Bsk in red and p-Bsk in green. Molecular mass markers are shown in lane 9. (D) $p$-Bsk protein levels in (A) were quantified and normalized relative to total JNK protein levels in (B) for each time point. The p-JNK:total JNK ratio at Omin LPS exposure was assigned a value of one and all other ratios are shown relative to this value. Treatment of S2 cells with LPS led to a transitory phosphorylation of Bsk with maximal Bsk phosphorylation at 5 mins.

times after exposure to PGN and simultaneously detected phospho-Bsk (p-Bsk) and total Bsk in the lysates by Western blot analysis using monoclonal anti phospho-JNK (p-JNK) and polyclonal anti-JNK antibodies. In contrast to standard ECL-based detection systems, we used secondary antibodies coupled to infrared dyes to detect the respective primary antibodies. Specifically, we chose infrared dyes with non-overlapping infrared emission spectra (Alexa-fluor 680 and Alexa-fluor 750) to distinguish total and phospho-Bsk (p-Bsk) on the Western blot using an infrared imager.

We observed a transitory increase in PGN-dependent Bsk phosphorylation, with maximal Bsk phosphorylation at five minutes post-PGN treatment and a return to basal $\mathrm{p}$ Bsk levels by sixty minutes (Fig. 1A). In comparison, total Bsk protein levels remained constant over the entire two hour period (Fig. 1B). We quantified the ratio of p-Bsk to total Bsk by measuring the relative intensities of the respective infrared fluorophores. We detected an eightfold increased in p-Bsk:total Bsk levels within five minutes 
of PGN exposure and the ratio returned to background levels by sixty minutes (Fig 1D). We note that our results are consistent with previous reports that Bsk is transiently phosphorylated through the Imd pathway upon exposure of S2 cells to PGN (21). In summary, we developed a Western Blot assay that allows detection and quantification of Bsk phosphorylation in response to PGN exposure in Drosophila S2 cell lysates.

The quantifiable Western blot assay described above relies on an infrared imaging system that has distinct advantages for relative quantification of protein samples. The infrared imaging system allows detection of multiple proteins on a single blot, without the need for stripping and re-probing. We used secondary antibodies coupled to distinct Alexa-fluor infrared dyes to detect the respective anti-total and p-Bsk primary antibodies. The use of infrared labels with distinct infrared emission spectra allows for the detection of two proteins on a single blot as the signals are separated into different infrared channels by the imaging system (Fig 1A-C).

Additionally, the infrared system allows relative quantification of protein levels. Traditional ECL detection relies on horseradish peroxidase-coupled secondary antibodies that convert luminol to light. The light is subsequently detected with X-ray film. The use of ECL does not allow for a reliable quantification of proteins because the production of light is only quantitatively linear over a small range of protein concentrations and the range varies with the exposure time of the blot to film. The use of film is also a drawback as it is difficult to detect saturation of the signal on the film. In contrast, the infrared system does not rely on an enzymatic reaction, but rather an infrared dye coupled to the secondary antibody. The signal to sample ratio is linear over a broad range of protein concentrations with infrared dye-labeled secondary antibodies. Additionally, the imaging software detects a saturated signal and the blot can be rescanned at lower excitation intensities to prevent signal saturation. These features allow for reliable quantification of proteins with the infrared detection system.

The infrared-based detection system relies on a control protein for relative quantification. In the assay above, we quantified $\mathrm{p}$-Bsk relative to total Bsk protein levels. However, we note that other proteins such as actin and tubulin would serve as relaible loading controls for quantification purposes. Housekeeping proteins are
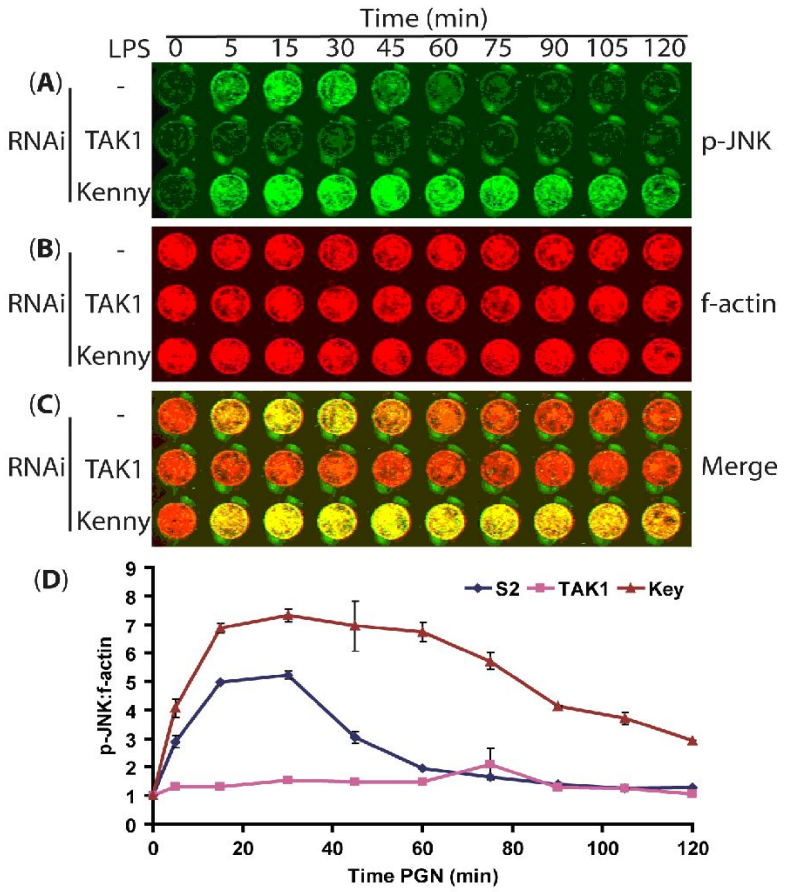

Fig. 2: In-Cell Quantification of Bsk Phosphorylation. (A,B) In-Cell quantitative analysis of S2 cells or S2 cells incubated with TAK1 or Kenny dsRNA and treated with LPS for the indicated periods. Cells were incubated with anti-p-JNK antibodies. The primary antibody was detected with an Alexa-fluor 750-labeled secondary antibody (A). Cellls were counterstained with Alexa-fluor 680-labeled phalloidin in (B). Panels A and B are falsecolored and merged in (C) with p-Bsk in green and f-actin in red. (D) p-Bsk protein levels from $(A)$ were quantified and normalized to f-actin levels in (B) for each time point. The p-JNK:total JNK ratio at Omin LPS exposure was assigned a value of one for each row and all other ratios are shown relative to this value. Error bars represent the standard error of two independent experiments. Treatment of S2 cells with LPS led to a transitory phosphorylation of Bsk, which was eliminated by dsRNA-mediated depletion of dTAK1 and enhanced and prolonged by dsRNA-mediated depletion of Kenny.

highly stable and their levels are relatively consistent from cell to cell. Indeed, we quantified p-Bsk relative to actin levels after PGN treatment and observed similar results to those described in Fig 1D (data not shown). While the assay described above is ideal for monitoring and quantifying alterations in p-Bsk levels, we note that the technique is applicable to a broad range of cellular events such as the dynamic alterations that accompany cell cycle progression, apoptosis and MAPK signaling.

Large Scale Analysis of JNK phosphorylation events in S2 cells

The Western blot protocol described above is an efficient method for analysis and quantification of a small number of samples. To perform experiments with large sample numbers, we developed a plate-based assay to monitor Bsk phosphorylation. Similar to the Western blot 
procedure described above, the assay uses infraredlabeled secondary antibodies to detect and quantify target proteins. Specifically, we grew S2 cells in 96 well tissue culture plates, stimulated the cells with LPS, fixed the cells directly in the plates and incubated with a monoclonal anti-p-JNK antibody. We detected p-Bsk with an Alexafluor 750 labeled secondary antibody that binds the anti-pBsk antibody. We counterstained the cells with Alexa-fluor 680-coupled phalloidin. Phalloidin binds filamentous actin (f-actin) and serves as a convenient control for cell number per well. As Alexa-fluor 680 and 750 dyes have non-overlapping infrared emission spectra, we quantified p-Bsk and f-actin levels in each well separately.

To determine relative Bsk phosphorylation, we measured the ratio of $\mathrm{p}$-Bsk to $\mathrm{f}$-actin at various times after PGN treatment. Consistent with the data presented in Fig. 1, we observed transient Bsk phosphorylation in response to exposure of S2 cells to PGN (Fig 2A). We detected maximal Bsk phosphorylation $15 \mathrm{~min}$ after exposure to PGN and p-JNK returned to basal levels within an additional $45 \mathrm{~min}$.

To validate the plate-based method, we determined the consequence of manipulating Imd pathway signaling for Bsk phosphorylation. dTAK1 is an essential MAPKKK for Bsk phosphorylation in response to PGN and loss of dTAK1 blocks PGN-dependent Bsk phosphorylation (14). Interestingly, we observed a complete block to PGNmediated Bsk phosphorylation in S2 cells treated with dsRNA targeting dTAK1 (Fig. 2A and D). Kenny is part of the IKK complex that activates Relish. A subset of Relresponsive transcripts contributes to Bsk inactivation and loss of Kenny leads to prolonged Bsk phosphorylation in S2 cells exposed to PGN (21). Similarly, we observed sustained Bsk phosphorylation in S2 cells treated with Kenny dsRNA and exposed to PGN (Fig. 2A and D). Thus, we conclude that the plate-based assay described above reproduces all known feature of Bsk activation by the Imd pathway and represents an ideal tool for direct quantification of Bsk activation.

There are several considerations to bear in mind regarding reagent choice in the plate-based assay. Cells must be adherent to avoid loss during the wash steps. To prevent cell loss, we used S2 cells grown in a serum-free medium. We found that $\mathrm{S} 2$ cells grown in serum-free medium are considerably more adherent than S2 cells grown in cell culture medium containing serum. In addition, it is
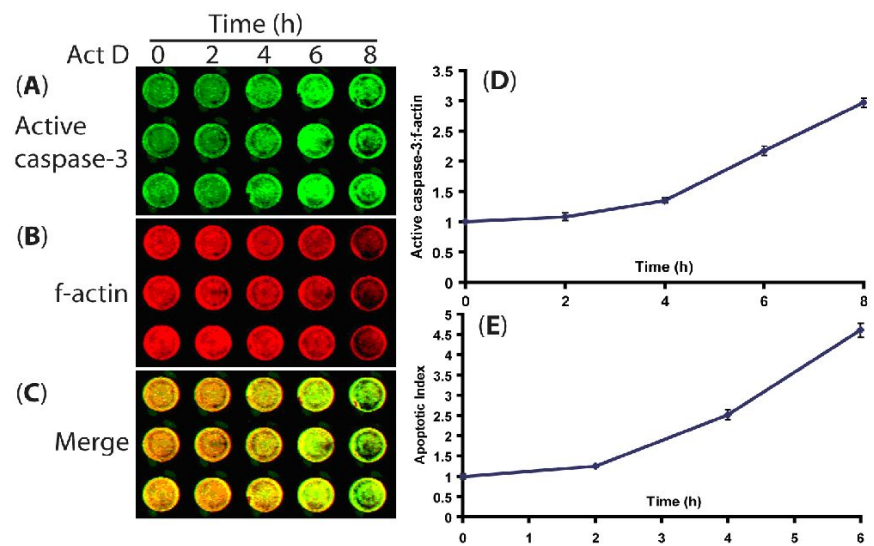

Fig. 3: Time Course of Caspase-3 activation in $\mathbf{S 2}$ cells. (A-C) InCell quantitative analysis of $\mathbf{S} 2$ cells treated for the indicated periods with Actinomycin D (Act D) to induce apoptosis. Each time point was measured in triplicate and stained for anti-active-caspase-3 (A) and f-actin (B). Panels $A$ and $B$ are false-colored and merged in (C) with active caspase- 3 in green and f-actin in red. (D) Active-caspase-3 protein levels from (A) were quantified and normalized to f-actin levels in (B) for each time point. The active caspase-3:f-actin ratio at Omin Actinomycin D exposure was assigned a value of one and all other ratios are shown relative to this value. Error bars represent the standard error of each independent measurement. Exposure of $S 2$ cells to Actinomycin D increased the relative levels of activecaspase-3 over time. (E) A stable S2 cell line that expresses Grim under control of the metallothionine promoter was incubated with $\mathrm{CuSO}_{4}$ for the indicated periods. The relative levels of apoptosis at each time point were determined by flow cytometry. The level of apoptosis at Omin copper exposure was assigned a value of one and all other ratios are shown relative to this value. Induction of Grim expression results in a steady increase in the levels of apoptosis.

important that the cell line used in the assay faithfully reproduces salient features of the event being assayed. We tested the Drosophila embryonic S2, S2R+ and KC167 cell lines and found the S2 cell line ideal for studying Bsk phosphorylation. In contrast, Bsk is not phosphorylated in S2R+ cells exposed to PGN and is only weakly phosphorylated in PGN-treated Kc167 cells (data not shown).

Unlike Western blots, where several bands are easily distinguished on a single blot, the plate-based assay only measures the total signal from each well of the plate, which makes the choice of antibody for the assay critical. The antibody should be specific for a single target and have low background plate binding. If the antibody binds more than one target, it will generate a signal that is not representative of the protein being studied. For our assay, we used an antibody that had only a single p-JNK band when used in a Western blot (Fig.1A). In addition, depletion of Bsk by RNAi resulted in a loss of the p-JNK signal in the plate-based assay (data not shown), confirming that the antibody is specific for p-Bsk in samples of fixed cells. We conclude that the protocol 
described above represents a robust method for direct quantification of Bsk activation in Drosophila tissue culture assays.

\section{Quantifiable Plate-Based Assay for Caspase Activation}

To determine the applicability of the In-Cell quantitative technique for quantification of other signaling events in S2 cells, we developed a plate-based assay for direct quantification of apoptosis. A key event in apoptotic progression in Drosophila is activation of effector and initiator caspases (24). To establish a plate-based assay to monitor caspase activation in Drosophila S2 cells, we exposed S2 cells in 96 well plates to the transcriptional inhibitor Actinomycin D. Actinomycin D treatment results in cytotoxic stress and concomitant induction of apoptotic death. We monitored caspase activation with a commercially available anti-active-caspase-3 antibody, which is a reliable reporter of apoptosis in Drosophila (25). The exact Drosophila epitope(s) detected by the anti-active caspase- 3 are unknown. However, this antibody is widely used as a measure of induction of apoptosis in Drosophila tissue culture and whole animal assays. We detected the anti-active-caspase-3 antibody with an Alexa-fluor 750labeled secondary antibody. We counterstained f-actin with Alexa-fluor 680-labeled phalloidin to control for cell numbers. We detected an increase in the active-caspase- 3 signal over the first six hours after Actinomycin D treatment, which decreased between six and eight hours (Fig. 3A). Similar to the active-caspase-3 signal, the f-actin signal declined at the eight-hour time point, however the signal was constant over the first six hours of Actinomycin $\mathrm{D}$ exposure (Fig. 3B). We believe the later loss of $\mathrm{f}$-actin staining is a result of apoptosis-induced detachment of cells from the substrate. Of note, we detected a steady increase in the ratio of active-caspase- 3 to f-actin during the entire eight-hour time course, indicating an overall increase in caspase activation on a per cell basis (Fig. 3D). We conclude that the assay described above provides a robust quantification of progression through apoptosis in S2 cells.

To compare the In Cell Western technique to alternative assays for detection of apoptosis, we used flow cytometry to monitor the induction of apoptosis in S2 cells that inducibly undergo apoptosis. To this end, we established a stable S2 cell line that expresses Grim under control of the copper-inducible metallothionine promoter. We did not detect expression of Grim in the absence of copper and noticed accumulation of Grim within one hour of copper addition (data not shown). We then measured the induction of apoptosis in cells expressing Grim at various times after addition of copper. Similar to our observations through In Cell Western analysis (e.g. Fig. 3D), we detected a steady accumulation of cells undergoing apoptosis upon induction of Grim expression (Fig. 3E). The detected rate of apoptotic induction is higher in cells expressing Grim and measured by flow cytometry than in cells exposed to Actinomycin D and measured by In Cell Western analysis. Importantly, flow cytometry-based quantification of apoptosis in S2 cells exposed to Actinomycin D revealed similar levels of apoptosis induction to Grim-expressing cells (data not shown). Thus, we conclude that flow cytometry is a more sensitive assay for the quantification of apoptosis than the In Cell Western procedure described here. Nonetheless, we note that both assays reliably report a steady accumulation in cells undergoing apoptosis.

The assay provides a valuable tool for the study of apoptotic signaling in Drosophila. However, like the platebased Bsk phosphorylation assay, the choice of reagents is critical. The selection of cells and the antibody used for the active-caspase- 3 assay require the same considerations as the Bsk phosphorylation assay. While we used f-actin as a measure for cell number, alternative stains such as btubulin are available for cell number quantification. We recommend that where feasible, users consider multiple measures of cell numbers as controls. Additionally, the time chosen to monitor caspase- 3 processing is a critical variable. Caspase-3 processing increases with time. However, as cells enter apoptosis they lose adherence and detach from the well. We performed a time course to determine the ideal exposure time of cells to Actinomycin $\mathrm{D}$ for our assay. We observed a significant increase in active-caspase-3 signal at the six hour time point (Fig. 3A). At the six-hour time point, cells remained adherent to the plates (Fig. 3B). The results indicate the best exposure time for S2 cells to Actinomycin D is six hours. Given that cells are less adherent by eight hours, we do not recommend quantifying apoptosis at this late stage. In summary, the plate-based assay outlined above provides an effective tool to monitor apoptotic events in Drosophila S2 cells efficiently in a large number of samples.

Numerous alternative immunofluorescence-based protocols exist for the quantification of signaling events in cells. For example, fluorescence-activated cell sorting (FACS) protocols exist for quantification of events as 
diverse as apoptosis and immune signaling in a broad range of cell lines. Cell sorters are commercially available with adaptors specifically designed for analysis of 96 and 384 well tissue culture plates, facilitating high-throughput analysis of large numbers of independent samples. In addition, cell sorters can distinguish a large number of individual fluorophores allowing multi-parameter analysis of individual samples. FACS-based assays are equally suited to adherent and non-adherent cell lines, while the protocol described in this manuscript is more suitable for evaluation of adherent cells. More recently, several high-content analysis instruments have been developed to allow statistical evaluation of complex cellular events in multi-well tissue culture plates. Such instruments typically capture and analyze immunofluouresce images according to parameters defined by the individual users. While costly and time consuming, these instruments facilitate the statistical analysis of complex cellular events and are ideally suited to multi-parameter analyses, particularly of subcellular criteria that require high-quality immunofluorescence imaging. In contrast, the In Cell Western assays described in this manuscript are restricted by several limitations. Specifically, all assays are restricted to fluorophores with infrared emission spectra, and are not suitable for multiparameter analyses. However, the instruments and reagents required are cost-effective and robust assays are easily developed for the quantification of "uncomplicated" on/off signaling events, making In-Cell analyses a viable alternative for a large number of highthroughput chemical or RNAi screens.

\section{ACKNOWLEDGMENTS}

MT-Grim DNA was generously provided by Dr. Kristin White. We are grateful to Tanja Hinck for assistance with flow cytometry. This work was funded by operating grants from the Canadian Institutes for Health Research and the Alberta Heritage Foundation for Medical Research. E.F. is a Scholar of the Alberta Heritage Foundation for Medical Research and holds a Canada Research Chair in Innate Immunity. The authors declare that no conflicts of interest exist.

\section{REFERENCES}

1. Beutler B. Innate immunity: an overview. Mol Immunol 2004;40:845-859.

2. Medzhitov R, Janeway CA Jr. Innate immunity: impact on the adaptive immune response.Curr Opin Immunol 1997;9:4-9.

3. Varfolomeev EE, Ashkenazi A. Tumor necrosis factor: an apoptosis JuNKie? Cell 2004;116:491-497.

4. Lemaitre B, Hoffmann J. The host defense of Drosophila melanogaster. Annu Rev Immunol 2007;25:697-743.

5. Lemaitre B, Nicolas E, Michaut L, Reichhart JM, Hoffmann JA. The dorsoventral regulatory gene cassette spatzle/Toll/cactus controls the potent antifungal response in Drosophila adults. Cell 1996;86:973-983.

6. Hoffmann JA. The immune response of Drosophila. Nature 2003;426:33-38.

7. Choe KM, Werner T, Stoven S, Hultmark D, Anderson KV. Requirement for a peptidoglycan recognition protein (PGRP) in Relish activation and antibacterial immune responses in Drosophila. Science 2002;296:359-362.

8. Gottar M, Gobert V, Michel T, Belvin M, Duyk G, et al. The Drosophila immune response against Gramnegative bacteria is mediated by a peptidoglycan recognition protein. Nature 2002;416:640-644.

9. Ramet M, Manfruelli P, Pearson A, Mathey-Prevot B, Ezekowitz RA. Functional genomic analysis of phagocytosis and identification of a Drosophila receptor for E. coli. Nature 2002;416:644-648.

10. Georgel P, Naitza S, Kappler C, Ferrandon D, Zachary $\mathrm{D}$, et al. Drosophila immune deficiency (IMD) is a death domain protein that activates antibacterial defense and can promote apoptosis. Dev Cell 2001;1:503-514.

11. Kleino A, Valanne S, Ulvila J, Kallio J, Myllymaki H, et al. Inhibitor of apoptosis 2 and TAK1-binding protein are components of the Drosophila Imd pathway. Embo J 2005;24:3423-3434.

12. Gesellchen V, Kuttenkeuler D, Steckel M, Pelte N, Boutros M. An RNA interference screen identifies Inhibitor of Apoptosis Protein 2 as a regulator of innate immune signalling in Drosophila. EMBO Rep 2005;6:979-984.

13. Vidal S, Khush RS, Leulier F, Tzou P, Nakamura M, et al. Mutations in the Drosophila dTAK1 gene reveal a conserved function for MAPKKKs in the control of rel/NF-kappaB-dependent innate immune responses. Genes Dev 2001;15: 1900-1912.

14. Silverman N, Zhou R, Erlich RL, Hunter M, Bernstein $\mathrm{E}$, et al. Immune activation of NF-kappaB and JNK requires Drosophila TAK1. J Biol Chem 2003;278:48928-48934.

15. Boutros M, Agaisse H, Perrimon N. Sequential 
Activation of Signaling Pathways during Innate Immune Responses in Drosophila. Dev Cell 2002;3:711-722.

16. Silverman N, Zhou R, Stoven S, Pandey N, Hultmark D, et al. A Drosophila IkappaB kinase complex required for Relish cleavage and antibacterial immunity. Genes Dev 2000;14:2461-2471.

17. Dushay MS, Asling B, Hultmark D. Origins of immunity: Relish, a compound Rel-like gene in the antibacterial defense of Drosophila. Proc Natl Acad Sci USA 1996;93: 10343-10347.

18. Wu LP, Anderson KV. Regulated nuclear import of Rel proteins in the Drosophila immune response. Nature 1998;392:93-97.

19. Stoven S, Silverman N, Junell A, Hedengren-Olcott M, Erturk D, et al. Caspase-mediated processing of the Drosophila NF-kappaB factor Relish. Proc Natl Acad Sci USA 2003;100:5991-5996.

20. Stoven S, Ando I, Kadalayil L, Engstrom Y, Hultmark D. Activation of the Drosophila NF-kappaB factor Relish by rapid endoproteolytic cleavage. EMBO Rep 2000;1:347-352.

21. Park JM, Brady H, Ruocco MG, Sun H, Williams D, et al. Targeting of TAK1 by the NF-kappa B protein Relish regulates the JNK-mediated immune response in Drosophila. Genes Dev 2004;18:584-594.

22. Foley E, O'Farrell PH. Functional Dissection of an Innate Immune Response by a Genome-Wide RNAi Screen. PLoS Biol 2004;2:E203.

23. Kaneko T, Goldman WE, Mellroth P, Steiner H, Fukase $\mathrm{K}$, et al. Monomeric and polymeric gram-negative peptidoglycan but not purified LPS stimulate the Drosophila IMD pathway. Immunity 2004;20:637-649.

24. Hay BA, Guo M. Caspase-Dependent Cell Death in Drosophila. Annu Rev Cell Dev Biol 2006;22:623-650.

25. Primrose DA, Chaudhry S, Johnson AG, Hrdlicka A, Schindler $\mathrm{A}$, et al. Interactions of DNR1 with the apoptotic machinery of Drosophila melanogaster. J Cell Sci 2007;120:1189-1199.

\section{PROTOCOLS}

In-Cell Western Protocol for the Detection of Active Bsk in S2 Cells

\section{Day 1}

- Add dsRNA at 10ug/well in $100 \mu \mathrm{l}$ serum-free growth media in a 96 well plate.

O rock to mix dsRNA for $5 \mathrm{~min}$

- Add $50 \mu \mathrm{l}$ of serum-deprived S2 cells at a concentration of 3 million cells $/ \mathrm{ml}$ in $60 \%$ conditioned medium to each well. The final cell concentration is $150,000 /$ well in $20 \%$ conditioned medium.

○ Briefly rock to evenly distribute cells

- Incubate plate at $25^{\circ} \mathrm{C}$ for 3 days in a humidified incubator.

\section{Day 4}

- Stimulate cells with LPS by adding $2 \mu 1 \mathrm{l} 100 \mathrm{x}$ LPS $(5 \mathrm{mg} / \mathrm{ml}$ ) to $48 \mu \mathrm{l}$ media and add $50 \mu \mathrm{l}$ per well (final concentration of $50 \mathrm{ug} / \mathrm{ml}$ LPS).

- Incubate cells with LPS for the appropriate time.

- Carefully remove media by inverting the plate once.

- Wash wells $1 x$ with $200 \mu l$ PBS per well.

- Add $150 \mu$ l fixing solution (PBS + 3.7\% formaldehyde) for $15 \mathrm{~min}$ at room temperature on the bench top (do not shake).

- remove fixative solution by inverting plate.

- Wash 96 well plate by submersion in PTX (PBS + 0.1\% Triton-X 100), wash for $6 \mathrm{~min}$ on the shaker.

$\circ$ remove solution by inverting plate.

O repeat wash a further 3 times.

- Add $150 \mu \mathrm{l}$ per well Li-COR Odyssey Blocking Buffer for 1hour.

o Remove blocking buffer.

- Add anti-Phospho-SAPK/JNK (T183/Y185) mouse monoclonal (Cell Signaling, 9255S).

- Add $30 \mu \mathrm{l}$ antibody to $6 \mathrm{ml}$ blocking buffer (1:200) and add $50 \mu \mathrm{l}$ per well. 
O Incubate overnight at $4^{\circ} \mathrm{C}$ shaking.

\section{Day 5}

- Wash plate by submersion in wash solution (PBS + 0.1\% Tween-20), wash for $6 \mathrm{~min}$ on the shaker

$\circ$ Remove solution by inversion

- Repeat 3 additional times

- Add secondary goat anti-mouse, and Alexa Fluor 680 phalloidin (Invitrogen)

$\circ$ Add $6 \mu$ l antibody (1:1000) and $6 \mu$ l phalliodin (1:1000) to $6 \mathrm{~mL}$ blocking buffer tween-20 (0.1\% tween-20)

$\circ$ Add $50 \mu \mathrm{l}$ per well and shake at room temperature in the dark for 1hour

- Wash 96 well plate by submersion in wash solution for $6 \mathrm{~min}$ on the shaker

○ Remove solution by inversion

- Repeat 3 additional times

- Rinse plate with $300 \mu \mathrm{l}$ PBS per well for $5 \mathrm{~min}$

O Repeat 1 time

0 Remove PBS and gently blot the plate on paper towel to remove excess moisture

○ Wash the back of plate with dsH2O and $95 \%$ ethanol before visualization

\section{Visualize}

- Place plate in Aerius Automated Infrared Imaging System

- Scan the plate at 800 and $700 \mathrm{~nm}$ simultaneously, using $200 \mu \mathrm{m}$ resolution, $3.0 \mathrm{~mm}$ focus offset, and an intensity setting of 9.5 for the $800 \mathrm{~nm}$ channel and 5.5 for the $700 \mathrm{~nm}$ channel. 